Conveni ent modul ar method for af fi nity I abel ing ( NoAL method) based on a cat al yt i c ami dat $i$ on

\begin{tabular}{|l|l|}
\hline 著者 & $\begin{array}{l}\text { Kuni shi ma Munet aka, Nakani shi Shui chi, N shi da } \\
\text { Ji n, Tanaka H r oyuki, Nor i saki Dai ki , H oki } \\
\text { Kazuhi to, Nonnt o Hi r oshi }\end{array}$ \\
\hline $\begin{array}{l}\text { j our nal or } \\
\text { publ i cat i on ti tl e }\end{array}$ & Chemi cal Commeni cat i ons \\
\hline vol une & 37 \\
\hline page range & $5597-5599$ \\
\hline year & $2009-01-01$ \\
\hline URL & ht t p: //hdl . handl e. net /2297/24283 \\
\hline
\end{tabular}




\title{
Convenient modular method for affinity labeling (MoAL method) based on a catalytic amidation $\dagger$
}

\author{
Munetaka Kunishima, ${ }^{* a c}$ Shuichi Nakanishi, ${ }^{a}$ Jin Nishida, ${ }^{b}$ Hiroyuki Tanaka, ${ }^{a}$ \\ Daiki Morisaki, ${ }^{a}$ Kazuhito Hioki ${ }^{b c}$ and Hiroshi Nomoto ${ }^{d}$
}

Received (in Cambridge, UK) 30th June 2009, Accepted 12th August 2009

First published as an Advance Article on the web 19th August 2009

DOI: $10.1039 / b 912908 a$

\begin{abstract}
A modular methodology for affinity labeling, in which three essential elements generally constituting affinity probes are prepared separately as individual molecules, has been developed based on a catalytic amidation.
\end{abstract}

Affinity labeling (AL) is a useful methodology for structural analysis of the binding site of biomolecules as well as for discovering target biomolecules of ligands or drugs. ${ }^{1-5}$ In general, affinity probes contain three essential elements in a single molecule (Fig. 1a): a ligand with specific affinity toward the target biomolecule; a labeling tag, such as an isotope or fluorescent moiety to facilitate the detection of the labeled biomolecules; and a reacting group to form a covalent bond at the binding site. Their inherent complexity presents several challenges to developing affinity probes: (1) design and synthesis of affinity probes are often difficult, (2) the binding activity of ligands toward the target biomolecules may be altered by introduction of the other elements, (3) fine tuning of affinity probes based on structure-activity relationships is not easy, (4) a labeling tag covalently introduced into the probe cannot be readily exchanged with others (low variation of labeling groups). In addition, the fact that the reacting group must be stable until the labeling experiment is undertaken, and that the labeling reaction must occur in an aqueous media present further difficulties in the design of affinity probes. Probably the most well-studied method for AL is photoaffinity labeling (PAL), in which a photo-reactive group (photophore) is employed as a reacting group, and labeling can take place by photo-irradiation. Although several good photophores have been developed over more than four decades, the main issues noted above remain inadequately addressed. ${ }^{5}$

A simple and elegant approach to the design and creation of affinity probes is the separation of the elements into individual molecules. ${ }^{6}$ For example, Hamachi and co-workers reported that a combination of sugar-tethered 4-dimethylaminopyridine and

${ }^{a}$ Faculty of Pharmaceutical Sciences, Institute of Medical, Pharmaceutical, and Health Sciences, Kanazawa University, Kakuma-machi, Kanazawa 920-1192, Japan.

E-mail: kunisima@p.kanazawa-u.ac.jp; Fax: (+81) 76-264-6201; Tel: $(+81)$ 76-264-6201

${ }^{b}$ Faculty of Pharmaceutical Sciences, Kobe Gakuin University, 1-1-3 Minatojima Chuo-ku, Kobe 655-8586, Japan

${ }^{c}$ Cooperative Research Center of Life Sciences, Kobe Gakuin University, 1-1-3 Minatojima Chuo-ku, Kobe 655-8586, Japan

${ }^{d}$ College of Pharmaceutical Sciences, Matsuyama University, 4-2 Bunkyo-cho, Matsuyama 790-8578, Japan

$\dagger$ Electronic supplementary information (ESI) available: Further experimental details and data. See DOI: 10.1039/b912908a an acyl donor possessing fluorescein were effective for targetselective labeling of lectins. ${ }^{6 a}$ Since the ligand and the labeling agent are separated into different molecules, synthesis and modification of ligand is much easier. We now report a convenient modular method for AL (MoAL), in which the above three essential elements are introduced as totally separate molecules (modules) (Fig. 1b).

We have already developed a novel catalytic dehydrocondensing reaction that proceeds in an aqueous solvent by using a combination of 2-chloro-4,6-dimethoxy-1,3,5-triazine (CDMT) and $N, N$-dimethylglycine esters as a catalyst. ${ }^{7}$ Thus, the novel MoAL method was designed based on this reaction. Because the main purpose of the present study is establishment of the methodology for the MoAL technique, we employed avidin and biotin as simple models for protein and ligand. As illustrated in Fig. 1c, biotin can be readily converted into a catalyst (ligand catalyst: $\mathbf{1}$ ) by introduction of $N, N$-dimethylglycine as a catalytic site. The catalytic site was connected to biotin with a linker of an appropriate length so that it could reach a carboxyl group at the protein surface of avidin. Cascade Blue ${ }^{\circledR}$ ethylenediamine, trisodium salt (CBA) was employed as a fluorescent primary amine. When avidin was treated with a mixture of $\mathbf{1}$ (ligand module), CBA (labeling module), and CDMT (reactive module), the carboxyl groups of aspartate or glutamate residues located at the protein surface in the vicinity of the biotin-binding pocket can specifically couple with CBA to give a labeled avidin. The activation of the carboxylates through the formation of an avidin/biotin complex will be accelerated by the proximity effect (proximity-accelerated reaction) even at a low concentration (mM order or less), ${ }^{2}$ at which a simple bimolecular reaction between an ammoniotriazine and a carboxylate is very slow. ${ }^{7}$

First, we examined the avidin-specific labeling in the presence of $\gamma$-globulins. A mixture of avidin and $\gamma$-globulins was treated with the ligand catalyst $\mathbf{1}$ at RT for $30 \mathrm{~min}$ followed by addition of CDMT and CBA, and the mixture was allowed to incubate for $8 \mathrm{~h}$. The proteins in the reaction mixture were separated by gel filtration chromatography, and each fraction was monitored by UV and fluorescence spectrophotometers. The elution profile of the proteins at $280 \mathrm{~nm}$ showed two peaks representing $\gamma$-globulins (the early peak) and avidin (the later peak), respectively (Fig. 2a). Strong fluorescence emissions at $423 \mathrm{~nm}$ were observed in the avidin fractions whereas the $\gamma$-globulins fractions showed only negligible signals. When a similar experiment was carried out using dimethylglycine ethyl ester $\mathbf{2}$ instead of $\mathbf{1}$, no significant 
a)

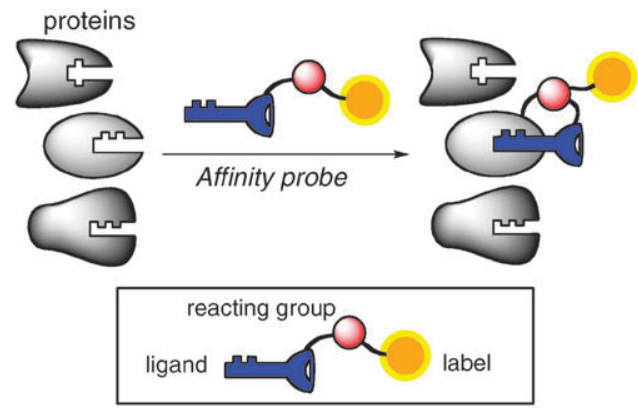

b)

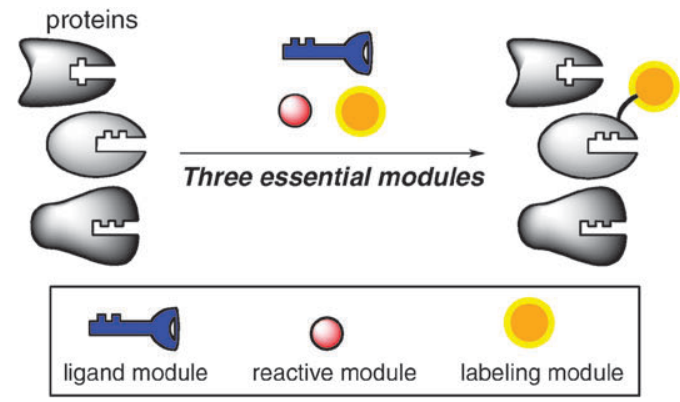

c)

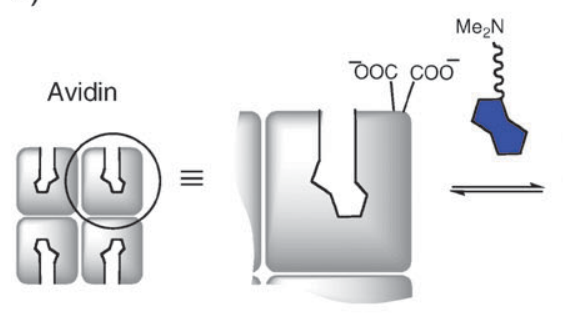

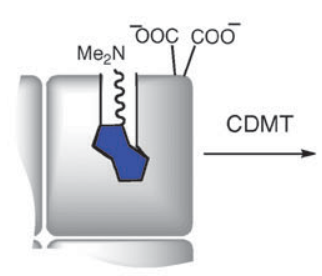

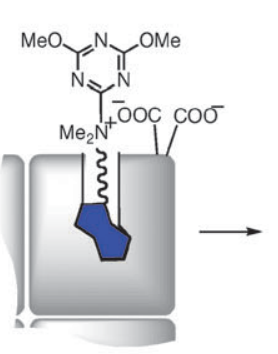

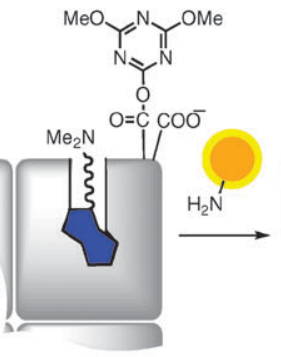

reactive module

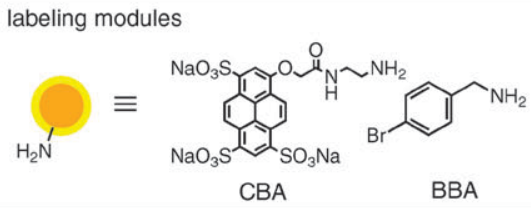

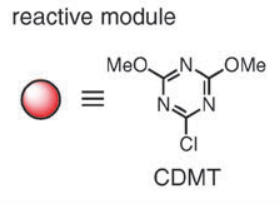
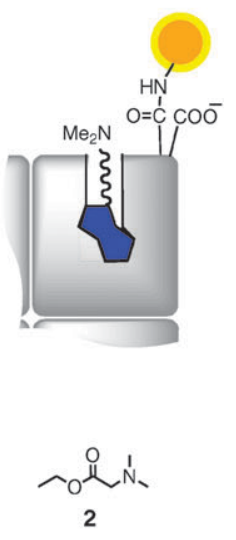

Fig. 1 (a) Conventional AL, in which three essential elements constitute a single affinity probe. (b) MoAL method, in which the three elements of the probe are totally separated as individual molecules (ligand, labeling and reactive modules). (c) Specific labeling of avidin with biotinylated ligand catalyst by the MoAL method based on a catalytic amide-forming reaction.

fluorescence emission was observed in the fractions of either protein (see Fig. S-3, ESI $\dagger$ ). The results indicate that the labeling of avidin occurred specifically with the biotin moiety.
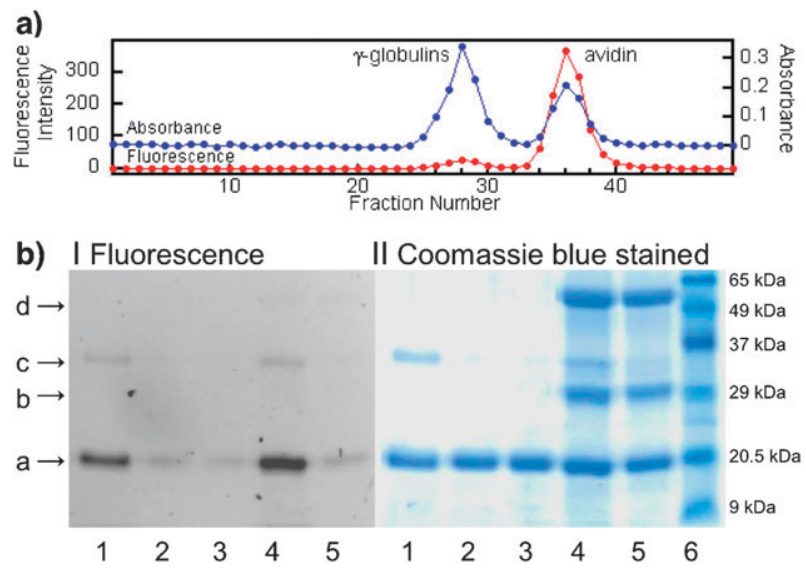

Fig. 2 (a) Elution profile of proteins. UV absorption at $280 \mathrm{~nm}$ and fluorescence intensity $\left(\lambda_{\mathrm{ex}} 399 \mathrm{~nm}, \lambda_{\mathrm{em}} 423 \mathrm{~nm}\right)$ are represented by blue and red lines, respectively. (b) SDS-PAGE analysis of specific labeling of avidin by the MoAL method. I: Fluorescence gel image, II: coomassie blue stained gel; lane 1: avidin $+\mathbf{1}$, lane 2: avidin $+\mathbf{1}+$ biotin (25 equiv.), lane 3: avidin $+\mathbf{2}$, lane 4 : avidin $+\gamma$-globulins $+\mathbf{1}$, lane 5: avidin $+\gamma$-globulins $+\mathbf{2}$, lane 6: molecular-weight marker. a: monomeric subunit of avidin, b: light chain of $\gamma$-globulins, c: dimeric subunits of avidin, $d$ : heavy chain of $\gamma$-globulins.
The labeling yields of proteins were determined by UV-visible spectral analysis. The yield of labeled avidin in the reaction with 1 for $8 \mathrm{~h}$ was found to be $169 \%$. Because avidin is a tetrameric protein composed of four identical subunits, each of which has a biotin-binding site, the observed yield over $100 \%$ would indicate that one or more subunits were labeled under the conditions. A control experiment using 2 as a catalyst gave only $10 \%$ yield of the labeled avidin under the same conditions. Similarly, $\gamma$-globulins were labeled with 1 in $11 \%$ yield. Thus, observed yields of about $10 \%(\sim 2.5 \%$ per a monomeric subunit) after $8 \mathrm{~h}$ are attributable to non-specific labeling by a simple intermolecular reaction of proteins and the condensing agents formed in a solution. The labeling reaction was completely inhibited by addition of an excess (25 equiv.) of biotin, indicating that the specific interaction between avidin and biotin is responsible for the labeling.

The labeled proteins were analyzed by SDS-PAGE (Fig. 2b). The fluorescence image of the gel indicated that avidin was significantly labeled with $\mathbf{1}$ (lanes 1,4 ) in contrast to the control experiments (lanes 2, 3, 5). No fluorescent signals were observed with $\gamma$-globulins (lanes 4, 5).

The SDS-PAGE experiments clearly indicate that the fluorescent amine (CBA) is covalently introduced into avidin by MoAL in a protein-specific manner. We carried out MS analysis to determine the modified amino acid. We originally employed CBA as a labeling agent because three sulfonato groups make CBA highly water soluble (and suppress the hydrophobic interaction with proteins), and because it is 


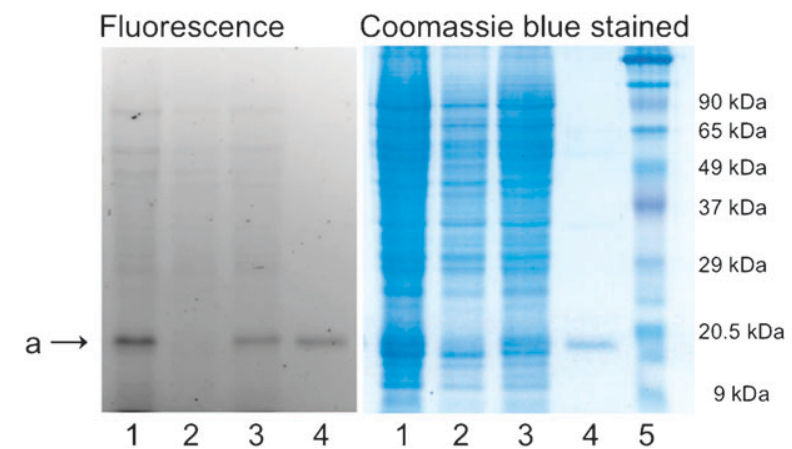

Fig. 3 SDS-PAGE analysis of specific labeling of avidin in the presence of PC12 cell lysates by the MoAL method using 1 and CBA. Lane 1: lysates including $2 \%$ avidin, lane 2 : lysates without addition of avidin, lane 3 : lysates including $4 \%$ avidin, lane 4 : avidin, lane 5: molecular-weight marker. a: monomeric subunit of avidin.

commercially available. The sulfonato groups, however, make MS analysis difficult, presumably due to their negative charge. In fact, no peak for CBA was observed by ESI-MS analysis under standard conditions. Taking advantage of the present MoAL method, in which a labeling agent is totally separated from the ligand, and therefore, readily changed with others, we employed 4-bromobenzylamine (BBA) instead of CBA as a labeling module. BBA facilitated MS analysis of the labeled amino acid because it gives characteristic peaks owing to the two isotopes of the bromine substituent (mixed isotope method). ${ }^{1,3,8}$

The peptide fragments obtained by in-gel digestion of the monomeric subunit of labeled avidin band on SDS-PAGE were directly analyzed by HPLC-ESI-MS/MS. As a result, Asp-108 was found to be specifically labeled with BBA. Interestingly, there are two other Asp residues (105 and 109) in the vicinity of the labeled Asp; no signal indicating their labeling was observed. Based on an X-ray structure, among 12 carboxyl groups (seven Asp and five Glu) in the monomeric subunit of avidin, Asp-108 seemed to occur at the most suitable position for labeling. Compared with conformations of the Asp-105 and 109 residues, the carboxyl group of Asp-108 is directed to the ligand catalyst 1 (see Fig. S-5, ESI $\dagger$ ).

Finally, in order to evaluate the potential generality of the MoAL method for discovering target proteins of ligands from biological samples, labeling experiments of avidin with $\mathbf{1}$ and CBA were conducted using cell lysates prepared from PC12 cells. Since avidin does not occur in the cells, we employed a sample prepared by addition of avidin $(2 \%$ or $4 \%$ of total proteins) to the lysates. As shown in Fig. 3, a significant specific labeling of avidin was observed in the presence of a number of different proteins.

In conclusion, the MoAL method using avidin/biotin as a model system appears to be effective for specific labeling of proteins. The most important feature of the MoAL is that the three modules (ligand, labeling tag, and reactive group) essential to affinity probes in AL methods are completely separated. Because ligands can be converted into catalysts by introducing $N, N$-dimethylglycine, design and synthesis of ligand catalysts would be much easier than with single molecule ALs. Various primary or secondary amines can be readily employed as a labeling agent using the modular method. In addition, neither photosensitive nor labile compounds need to be utilized, in marked contrast to PAL methods. Thus, the MoAL method is quite practical.

Nucleophilic amino acid residues such as Cys, His, Lys, Arg, Ser, and Tyr have been shown to be accessible as target amino acids in bioconjugation techniques for proteinmodification while modification methods for Asp and Glu are very limited. ${ }^{9}$ Thus, another advantage of the MoAL method is the fact that the carboxyl group is targeted for the modification. Because of significantly high relative abundances of Asp and Glu (more than 10\% in total in genome-wide analysis), ${ }^{10}$ and therefore the high frequency of carboxyl groups on a hydrophilic protein surface, the MoAL method should be widely applicable to a variety of proteins.

We thank Mr N. Iwasaki, Bruker Daltonics K.K. for MS analysis, and Accelrys K.K. for preparing a 3D structure of the complex. This work was supported partially by a Grantin-Aid for Science Research (No. 20390007) from the Ministry of Education, Culture, Sports, Science and Technology, Japan.

\section{Notes and references}

1 A. Sinz, Angew. Chem., Int. Ed., 2007, 46, 660.

2 (a) K. Levitsky, M. D. Boersma, C. J. Ciolli and P. J. Belshaw, ChemBioChem, 2005, 6, 890; (b) C. J. Krusemark and P. J. Belshaw, Org. Biomol. Chem., 2007, 5, 2201; (c) K. Levitsky, C. J. Ciolli and P. J. Belshaw, Org. Lett., 2003, 5, 693; (d) K. Tanaka, Y. Fujii and K. Fukase, ChemBioChem, 2008, 9, 2392.

3 S. M. Lamos, C. J. Krusemark, C. J. McGee, M. Scalf, L. M. Smith and P. J. Belshaw, Angew. Chem., Int. Ed., 2006, 45, 4329.

4 G. Chen, A. Heim, D. Riether, D. Yee, Y. Milgrom, M. A. Gawinowicz and D. Sames, J. Am. Chem. Soc., 2003, 125, 8130 .

5 (a) M. Hashimoto and Y. Hatanaka, Eur. J. Org. Chem., 2008, 2513; (b) E. L. Vodovozova, Biochemistry (Moscow), 2007, 72, 1; (c) D. Robinette, N. Neamati, K. B. Tomer and C. H. Borchers, Expert Review of Proteomics, 2006, 3, 399; (d) F. Kotzyba-Hibert, I. Kapfer and M. Goeldner, Angew. Chem., Int. Ed. Engl., 1995, 34, 1296; (e) S. A. Fleming, Tetrahedron, 1995, 51, 12479.

6 (a) Y. Koshi, E. Nakata, M. Miyagawa, S. Tsukiji, T. Ogawa and I. Hamachi, J. Am. Chem. Soc., 2008, 130, 245; (b) H. Wakabayashi, M. Miyagawa, Y. Koshi, Y. Takaoka, S. Tsukiji and I. Hamachi, Chem.-Asian J., 2008, 3, 1134; (c) T. Mayer and M. E. Maier, Eur. J. Org. Chem., 2007, 4711; (d) W. G. Lewis, L. G. Green, F. Grynszpan, Z. Radic, P. R. Carlier, P. Taylor, M. G. Finn and K. B. Sharpless, Angew. Chem., Int. Ed., 2002, 41, 1053; (e) S. Tsukiji, M. Miyagawa, Y. Takaoka, T. Tamura and I. Hamachi, Nat. Chem. Biol., 2009, 5, 341.

7 (a) M. Kunishima, K. Yoshimura, H. Morigaki, R. Kawamata, K. Terao and S. Tani, J. Am. Chem. Soc., 2001, 123, 10760; (b) M. Kunishima, H. Imada, K. Kikuchi, K. Hioki, J. Nishida and S. Tani, Angew. Chem., Int. Ed., 2005, 44, 7254.

8 (a) H. Li, Y. Liu, K. Fang and K. Nakanishi, Chem. Commun., 1999, 365; (b) M. Daghish, L. Hennig, M. Findeisen, S. Giesa, F. Schumer, H. Hennig, A. G. Beck-Sickinger and P. Welzel, Angew. Chem., Int. Ed., 2002, 41, 2293.

9 (a) G. T. Hermanson, Bioconjugate Techniques, Academic Press, San Diego, 1996; (b) I. S. Carrico, Chem. Soc. Rev., 2008, 37, 1423.

10 G. A. C. Singer and D. A. Hickey, Mol. Biol. Evol., 2000, 17, 1581. 